
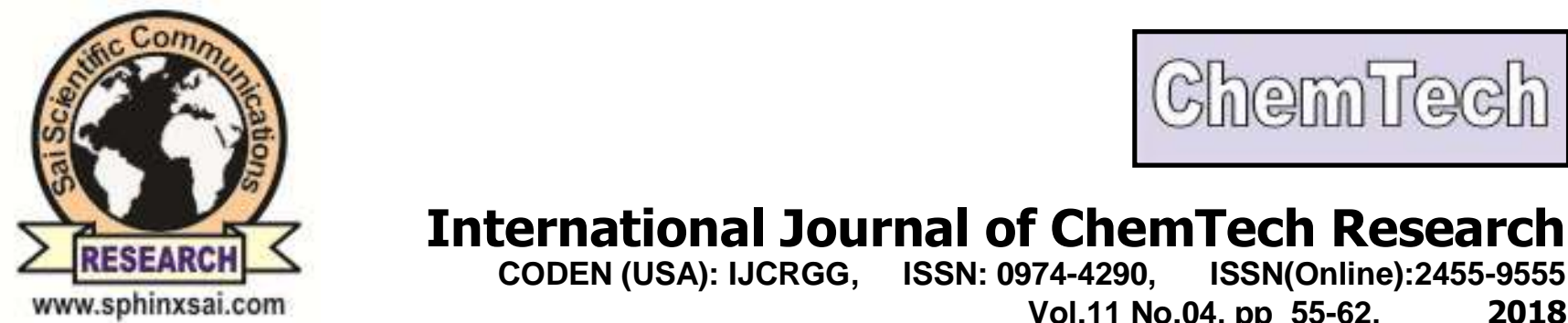

International Journal of ChemTech Research CODEN (USA): IJCRGG, ISSN: 0974-4290, ISSN(Online):2455-9555

Vol.11 No.04, pp 55-62,

2018

\title{
Bluetooth Based Automatic Floor Cleaning System
}

\author{
C.R.Balamurugan*, P.Kirubha, S.ArunKanna, E.R.Hariprasath, C.Anupriya
}

Department of EEE/Karpagam College of Engineering/Coimbatore, India

\begin{abstract}
It's a wireless Bluetooth control floor cleaning machine and will be amazed at the simplicity and effectiveness of the idea. It's basically DC motors wired in a wheeled plastic container with a cleaning solution placed on top and a scrub attached in the bottom through one of the motors. The brush cleans the floor and dried with aid of CPU fan. Anybody can operate this machine easily. Hence it is very useful in hospitals, houses, etc. Bluetooth module is used for controlling the entire system with help of remote or mobile. There is an application in mobile for Bluetooth connection between the system and mobile. By using Bluetooth module we can direct and turn the system as the user needs. It works great and controlled manually based on the user convenience. Definitely makes cleaning easier and merrier while enabling anyone to build something rather than buy. In modern days interior decorations are becoming an important role in our life. Cleaning of floor is a very important one for our health and reduces the man power requirement. Hence our project is very useful in our day to day life.

Keywords : Automatic; floor cleaning machine; bluetooth; prototype
\end{abstract}

\section{Introduction}

This paper deals with the designing and fabrication of Floor cleaning Machine. The aim of this work is to develop and modernized process for cleaning the floor with wet and dry. It is very useful for cleaning the floors. It can be used wet and dry. Floor cleaning system is very much useful in cleaning floors in hospitals, houses, auditorium, shops, computer centers etc; it is very simple in construction and eas y to operate. Anybody can operate this machine easily. It consist of moisture cotton brush, the brush cleans the floor and dried with aid of small blower. Hence it is very useful in hospitals, houses, etc. The time taken for cleaning is very less and the cost is also very less. Maintenance cost is less. Much type of machines is widely used for this purpose. But they are working under different principles and the cost is also very high. In this paper a very simple drive mechanism and easy to operate any persons. The size of the machine is also portable, so we can transfer from one place to other place very easily. The floor cleaning machine is simple, modern house holding device; even children can also operate it easily with safety. It is very important one for each and every houses and hospitals etc.

\section{Concept Description}

With just materials you can readily find at home, here's a very creative and smart build that can take over the chore related to floor cleaning. It's a wireless floor cleaning machine controlled by bluetooth and

C.R.Balamurugan et al /International Journal of ChemTech Research, 2018,11(04): 55-62.

DOI : http://dx.doi.org/10.20902/IJCTR.2018.110406 
you'll be amazed at the simplicity and effectiveness of the idea. It's basically DC motors wired in a wheeled plastic container with a cleaning solution placed on top and a scrub attached in the bottom through one of the motors. It works great and seeing the mechanism is like seeing a robot assistant on duty. Definitely makes cleaning easier and merrier while enabling anyone to build something rather than buy.

Wireless and radio technologies have been at the heart of many disruptive businesses and activities. The people of today want hassle free and compact products, which can be used at any time or at any place. Wireless is the possible solution to meeting the needs or wants of the society. This project will discuss the emerging wireless technology such as Bluetooth. This project gives an in depth look on how the different technologies are being used today. By doing this, project will discuss how Bluetooth technology works, its origin, context, and implementation.

\section{Block Diagram}

The cleaning liquid is mixed in proper proportion and it is poured into the reservoir through the top way. The cleaning liquid is poured until the tanks are filled. Actuating the lever opens the valve. Cleaning liquid from the tank spills on the ground. After the required amount is delivered, the lever is released and the machine is switched ON. The brush gets drive from motor through pulleys and belts. The brush applies pressure on the floor, when the adjuster rod is unscrewed and the handle is moved in the required direction to clean the sufficient area. Again the lever is operated to supply cleaning liquid whenever required. This process is repeated, so floor is cleaned well. The cotton brush prevents the damages on the mosaic, marble floors and gives smooth surface finish and shining. This project is fully unified for cleaning applications. It features the requirements needed for floor cleaning such as water supply, scrub, and fan. It is a wheeled type machine with a movement control. This floor cleaning machine is comprised of several DC motors that drives the wheels and rotating objects for the scrub. Wiring of the motors are properly designed that the wheels are set up considering the control is from two dual two way switches. A pushbutton is also set as ON/OFF switch of the rotating objects as scrubs. Plastic pipes are also designed in which it has holes and gat valve that manages the release of cleaning liquid on the floor. It has two CPU fan after the scrubs used for drying of the scrubbed floor area. The machine is wired using LAN wires connected to its controller while the controller has the connection of the $12 \mathrm{~V}$ supply.

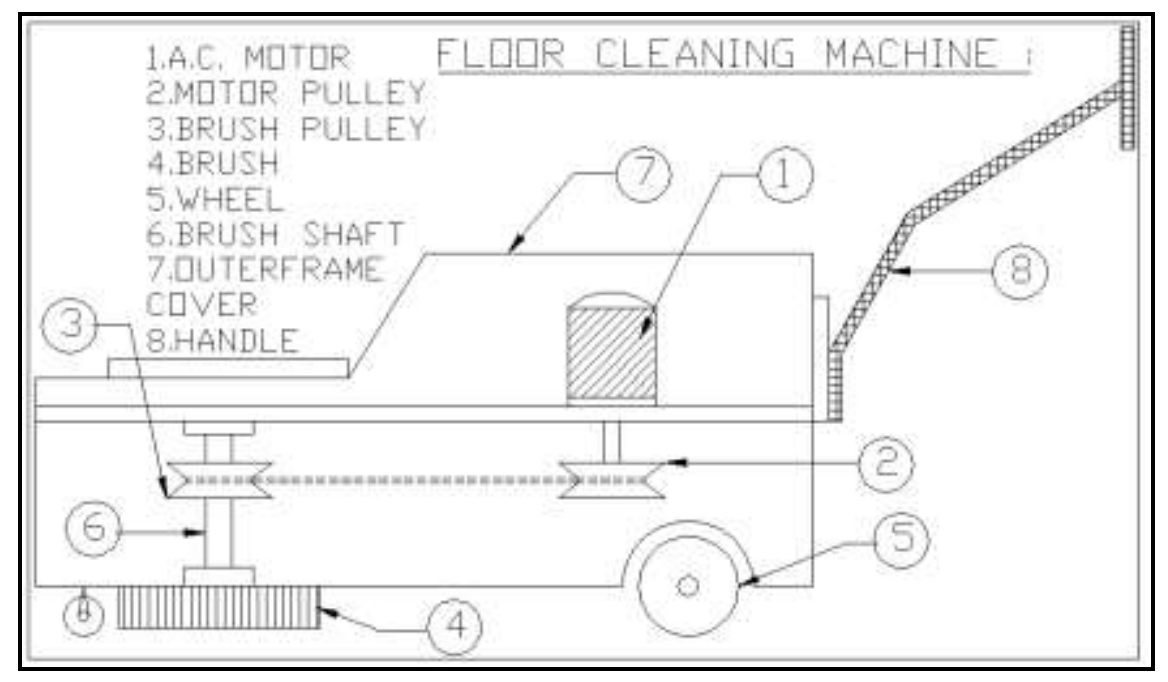

Fig. 1. Block diagram of proposed model

This paper is applicable for several floor cleaning activities. Considering the area and access of the floor to be cleaned, this floor cleaning machine is able to handle a load of cleaning activity. 


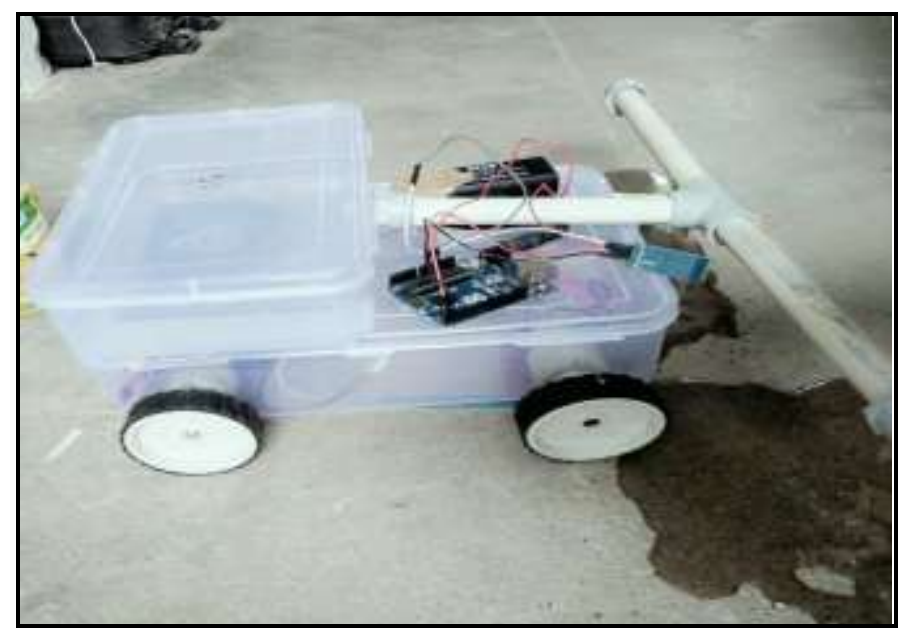

Fig. 2. Hardware Prototype

\section{a) DC gear motor}

In this work, gear DC motor is used. A DC motor is not the same as a "gear motor" - a "gear motor" may be an AC or DC motor coupled with a gearbox or transmission. A gear motor adds mechanical gears to alter the speed/torque of the motor for an application. Usually such an addition is to reduce speed and increase torque. Geared DC motors can be defined as an extension of DC motor. A geared DC Motor has a gear assembly attached to the motor. The speed of motor is counted in terms of rotations of the shaft per minute and is termed as RPM .The gear assembly helps in increasing the torque and reducing the speed. Using the correct combination of gears in a gear motor, its speed can be reduced to any desirable figure. This concept where gears reduce the speed of the vehicle but increase its torque is known as gear reduction. This Insight will explore all the minor and major details that make the gear head and hence the working of geared DC motor.

\section{b) External Structure}

Gear motors are an all-in-one combination of an electric motor and gears or a gearbox. A gear motor simplifies combining a motor with a gear reducer system. Gears are used with motors to lower the motor's speed while increasing the output torque. A simple electric motor can be built using a coil of wire that is free to rotate between two opposite magnetic poles. When an electric current flows through the coil, the coil experiences a force and moves. At the first sight, the external structure of a DC geared motor looks as a straight expansion over the simple DC ones. The lateral view of the motor shows the outer protrudes of the gear head. A nut is placed near the shaft which helps in mounting the motor to the other parts of the assembly.

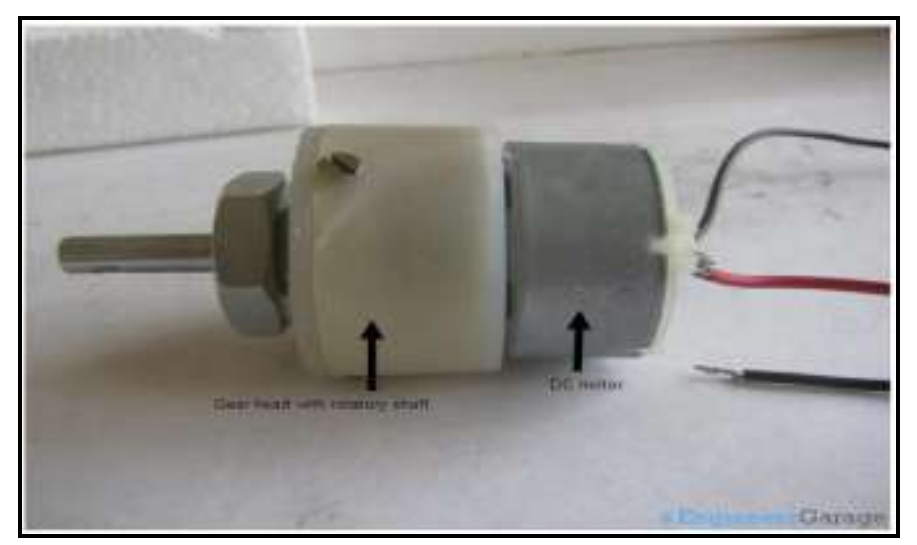

Fig. 3. Side View of Gear Motor 


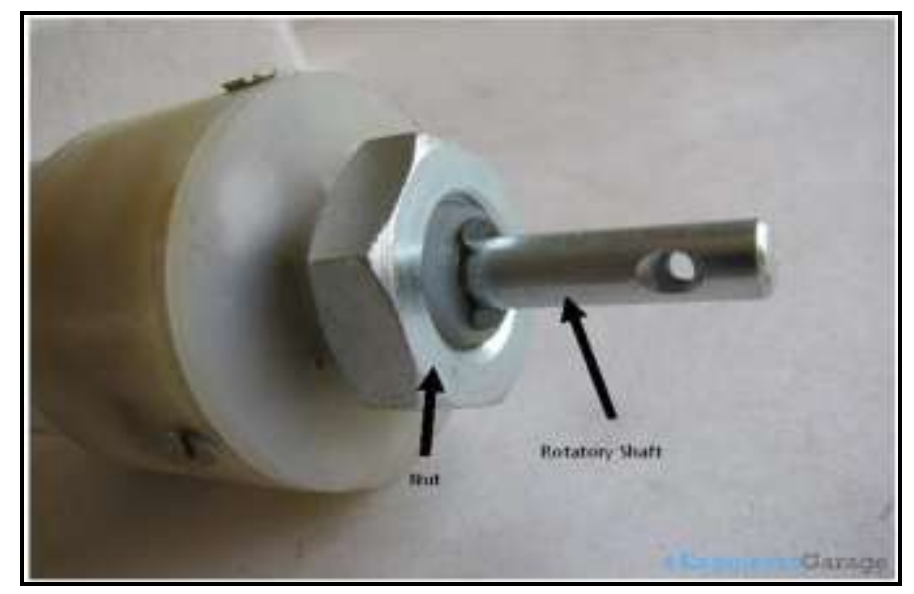

Fig. 4. Rear View of Gear Motor

Also, an internally threaded hole is there on the shaft to allow attachments or extensions such as wheel to be attached to the motor.

\section{Bluetooth Module}

Bluetooth wireless technology is a $2.4 \mathrm{GHz}$ ISM-band open industry standard for short range wireless communication, which is capable of voice and data transfer (up to $723 \mathrm{kbps}$ data transfer in ACL link or up to 3 simultaneous voice connections in SCO links in a piconet).In this document, the SEMCO-Bluetooth Modules are presented, which consists of RF, baseband and link manager protocol together with Host Controller Interface(HCI) / HCI-UART functionality implemented according to the Bluetooth specification version 1.1.SEMCO-Bluetoth Modules are designed for use as an universal Bluetooth module compliant for the Bluetooth specification version 1.1, which can be applied to hand-held phones, PDAs, headsets, PCs and PCperipherals, et cetera for wireless voice and data communication. Most of us have experienced trying to figure out what cable goes where, and ended up tangled in the details. Bluetooth essentially aims to fix this problem. This is considered a cable-replacement technology.

\section{a) Short Range Wireless}

There are many short-range digital communications among computing and communications devices; today much of that communication takes place over cables. These cables connect to multitude of devices using a wide variety of connectors with many combinations of shapes, sizes and number of pins; this plethora of cables can become quite burdensome to user. With Bluetooth technology, this technology can communicate without wires over a single air interface, using radio waves to transmit and receive data. Bluetooth wireless technology is specifically designed for short-range communications; one result of this very low power consumption, making the technology well suited for use with small, portable personal devices that typically are powered by batteries.

\section{b) Voice and Data}

Voice appliances such as mobile telephones are also used for data applications such as information access or browsing. Trough voice recognition, computers can be controlled by voice, and through voice synthesis, computers can produce audio output in addition to visual output. Some wireless communication technologies are designed to carry only voice while others handle only data traffic. Bluetooth wireless communication makes provisions for both voice and data, and thus it is an ideal technology for unifying these worlds by enabling all sorts of devices to communicate using either or both of these content types Bluetooth is split into two sections: Bluetooth Specification and Bluetooth Profile. The Specification describes how the technology works (i.e. the Bluetooth protocol architecture). The Profiles describe how the technology is used (i.e. how different parts of the specification can be used to fulfill a desired function for a Bluetooth device).

\section{c) Technical Features}

Bluetooth operates in the unlicensed $2.4 \mathrm{GHz}$ Industrial Scientific Medical (ISM) frequency band as shown in Figure 4 below. It uses 79 channels between $2.402 \mathrm{GHz}$ to $2.480 \mathrm{GHz}$. It uses power of $1 \mathrm{~mW}$ to 
$100 \mathrm{~mW}$. The nominal range is 10 meters, but can be extended to more than 100 meters by increasing the transmission power to $100 \mathrm{~mW}$. The gross data rate is $1 \mathrm{Mbps}$. Bluetooth uses a combination of packet and circuit switching technologies for transmission.

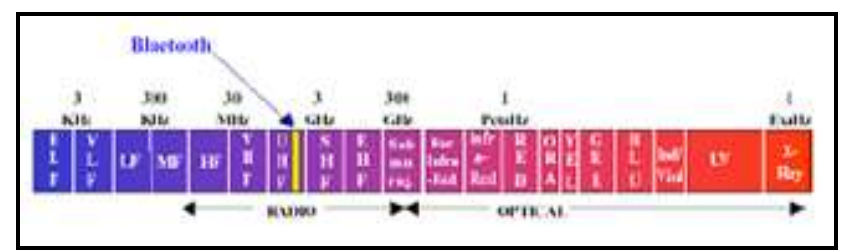

Fig. 5. ISM Band

While developing the specification, high emphasis was placed on developing a design that would enable single chip CMOS implementations, thereby reducing cost, power consumption, and the chip size required for implementation in mobile devices. A single chip CMOS is already available from multiple manufacturers. This device contains the RF radio, baseband controller and microprocessor on a single CMOS integrated circuit.

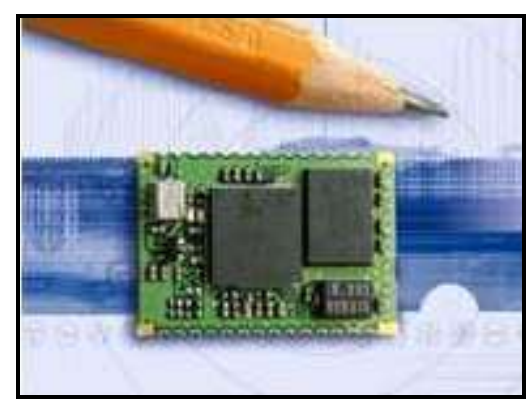

Fig. 6. Single Chip CMOS

\section{d) Access Technology}

Since Bluetooth operates in the unlicensed ISM band that is also used by other devices such as 802.11 networks, baby monitors, garage door openers, microwave ovens etc, there is possibility of interference (Helsinki University of Technology, 2000). To avoid interference, Bluetooth uses an access technology called Frequency Hop Spread Spectrum (FHSS). The band where Bluetooth operates is divided into 79 channels of 1 $\mathrm{MHz}$ each. Each channel is divided into time slots consisting of $625 \mu \mathrm{sec}$ in length. After a packet is sent on one frequency, both the devices retune their radios to a different frequency, effectively hopping at a rate of 1600 hops per second through different time slots. In this way, Bluetooth devices use the entire ISM band and if a transmission is compromised by interference on one channel, the retransmission will always be on a different (hopefully clear) channel. Bluetooth Network consists of a Personal Area Network or a piconet which contains a minimum of 2 to maximum of 8 bluetooth peer devices- Usually a single master and upto 7 slaves. A master is the device which initiates communication with other devices. The master device governs the communications link and traffic between itself and the slave devices associated with it. A slave device is the device that responds to the master device. Slave devices are required to synchronize their transmit/receive timing with that of the masters. In addition, transmissions by slave devices are governed by the master device (i.e., the master device dictates when a slave device may transmit). Specifically, a slave may only begin its transmissions in a time slot immediately following the time slot in which it was addressed by the master, or in a time slot explicitly reserved for use by the slave device. The frequency hopping sequence is defined by the Bluetooth device address (BD_ADDR) of the master device. The master device first sends a radio signal asking for response from the particular slave devices within the range of addresses. The slaves respond and synchronize their hop frequency as well as clock with that of the master device. Scatter nets are created when a device becomes an active member of more than one piconet. Essentially, the adjoining device shares its time slots among the different piconets.

\section{Arduino Uno}

The Arduino Uno is a microcontroller board based on the ATmega328. It has 14 digital input/output pins (of which 6 can be used as PWM outputs), 6 analog inputs, a $16 \mathrm{MHz}$ crystal oscillator, a USB connection, 
a power jack, an ICSP header, and a reset button. It contains everything needed to support the microcontroller; simply connect it to a computer with a USB cable or power it with a AC-to-DC adapter or battery to get started. The Uno differs from all preceding boards in that it does not use the FTDI USB-to-serial driver chip. Instead, it features the Atmega16U2 (Atmega8U2 up to version R2) programmed as a USB-to-serial converter. Revision 2 of the Uno board has a resistor pulling the $8 \mathrm{U} 2 \mathrm{HWB}$ line to ground, making it easier to put into DFU mode.

Revision 3 of the board has the following new features:

- pinout: added SDA and SCL pins that are near to the AREF pin and two other new pins placed near to the RESET pin, the IOREF that allow the shields to adapt to the voltage provided from the board. In future, shields will be compatible both with the board that use the AVR, which operate with $5 \mathrm{~V}$ and with the Arduino Due that operate with $3.3 \mathrm{~V}$. The second one is a not connected pin, that is reserved for future purposes.

- Stronger RESET circuit.

- Atmega $16 \mathrm{U} 2$ replace the $8 \mathrm{U} 2$.

"Uno" means one in Italian and is named to mark the upcoming release of Arduino 1.0. The Uno and version 1.0 will be the reference versions of Arduino, moving forward. The Uno is the latest in a series of USB Arduino boards, and the reference model for the Arduino platform; for a comparison with previous versions, see the index of Arduino boards. Microcontroller ATmega328 Operating Voltage 5V Input Voltage (recommended) 7-12V Input Voltage (limits) 6-20V Digital I/O Pins 14 (of which 6 provide PWM output) Analog Input Pins 6 DC Current per I/O Pin $40 \mathrm{~mA}$ DC Current for 3.3V Pin $50 \mathrm{~mA}$ Flash Memory $32 \mathrm{~KB}$ (ATmega328) of which $0.5 \mathrm{~KB}$ used by bootloader SRAM 2 KB (ATmega328) EEPROM 1 KB (ATmega328) Clock Speed $16 \mathrm{MHz}$.

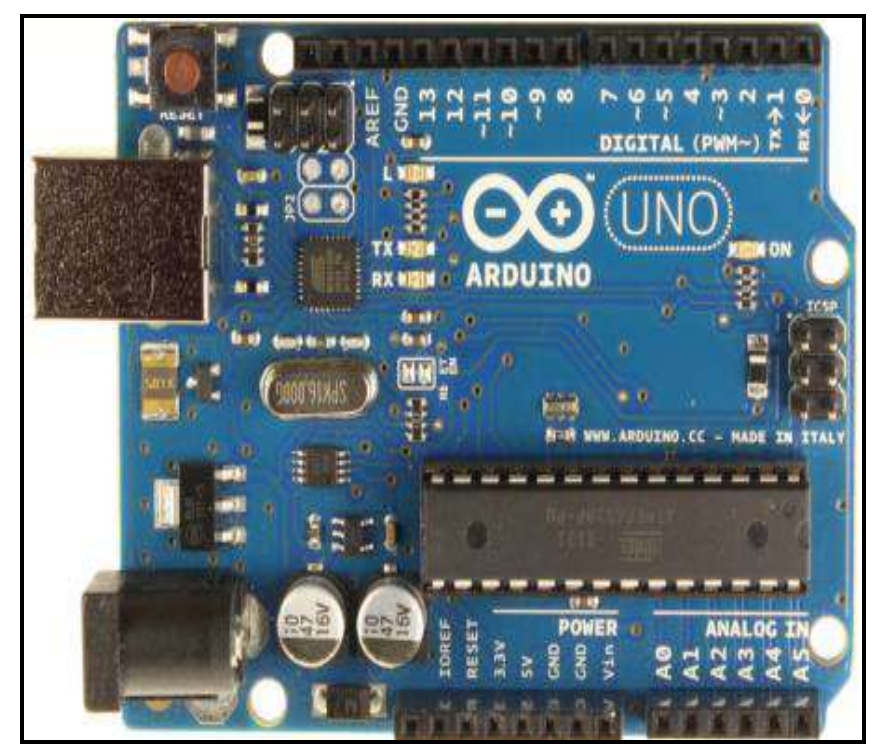

Fig. 7. Arduino Uno Board

\section{a) Power Supply}

The Arduino Uno can be powered via the USB connection or with an external power supply. The power source is selected automatically. External (non-USB) power can come either from an AC-to-DC adapter (wallwart) or battery. The adapter can be connected by plugging a $2.1 \mathrm{~mm}$ center-positive plug into the board's power jack. Leads from a battery can be inserted in the Gnd and Vin pin headers of the POWER connector. The board can operate on an external supply of 6 to 20 volts. If supplied with less than $7 \mathrm{~V}$, however, the $5 \mathrm{~V}$ pin may supply less than five volts and the board may be unstable. If using more than $12 \mathrm{~V}$, the voltage regulator may overheat and damage the board. The recommended range is 7 to 12 volts.

The power pins are as follows: 
- VIN. The input voltage to the Arduino board when it's using an external power source (as opposed to 5 volts from the USB connection or other regulated power source). You can supply voltage through this pin, or, if supplying voltage via the power jack, access it through this pin.

- $5 \mathrm{~V}$. The regulated power supply used to power the microcontroller and other components on the board. This can come either from VIN via an on-board regulator, or be supplied by USB or another regulated 5V supply.

- 3V3. A 3.3 volt supply generated by the on-board regulator. Maximum current draw is $50 \mathrm{~mA}$.

- GND. Ground pins.

\section{b) Input and Output}

Each of the 14 digital pins on the Uno can be used as an input or output, using pin Mode(), digital Write(), and digital Read() functions. They operate at 5 volts. Each pin can provide or receive a maximum of 40 $\mathrm{mA}$ and has an internal pull-up resistor (disconnected by default) of 20-50 k Ohms. In addition, some pins have specialized functions:

- Serial: 0 (RX) and 1 (TX). Used to receive (RX) and transmit (TX) TTL serial data. These pins are connected to the corresponding pins of the ATmega8U2 USB-to-TTL Serial chip.

External Interrupts: 2 and 3. These pins can be configured to trigger an interrupt on a low value, a rising or falling edge, or a change in value. See the attach Interrupt() function for details.

- PWM: 3, 5, 6, 9, 10, and 11. Provide 8-bit PWM output with the analog Write() function.

- SPI: 10 (SS), 11 (MOSI), 12 (MISO), 13 (SCK). These pins support SPI communication using the SPI library.

- LED: 13. There is a built-in LED connected to digital pin 13. When the pin is HIGH value, the LED is on, when the pin is LOW, it's off.

The Uno has 6 analog inputs, labeled A0 through A5, each of which provide 10 bits of resolution (i.e. 1024 different values). By default they measure from ground to 5 volts, though is it possible to change the upper end of their range using the AREF pin and the analog Reference() function. Additionally, some pins have specialized functionality: TWI: A4 or SDA pin and A5 or SCL pin. Support TWI communication using the Wire library. There are a couple of other pins on the board:

- AREF: Reference voltage for the analog inputs. Used with analog Reference().

- Reset: Bring this line LOW to reset the microcontroller. Typically used to add a reset button to shields which block the one on the board.

See also the mapping between Arduino pins and ATmega328 ports. The mapping for the Atmega8, 168, and 328 is identical.

\section{c) Communication}

The Arduino Uno has a number of facilities for communicating with a computer, another Arduino, or other microcontrollers. The ATmega328 provides UART TTL $(5 \mathrm{~V})$ serial communication, which is available on digital pins 0 (RX) and 1 (TX). An ATmega16U2 on the board channels this serial communication over USB and appears as a virtual com port to software on the computer. The '16U2 firmware uses the standard USB COM drivers, and no external driver is needed. However, on Windows, a .inf file is required. The Arduino software includes a serial monitor which allows simple textual data to be sent to and from the Arduino board. The RX and TX LEDs on the board will flash when data is being transmitted via the USB-to-serial chip and USB connection to the computer (but not for serial communication on pins 0 and 1). A Software Serial library allows for serial communication on any of the Uno's digital pins. The UNO also supports $\mathrm{I}^{2} \mathrm{C}$ (TWI) and SPI communication. The Arduino software includes a Wire library to simplify use of the $\mathrm{I}^{2} \mathrm{C}$ bus; see the documentation for details. For SPI communication, use the SPI library.

\section{Software For Interfacing Arduino And Bluetooth}

char data $=0$; //Variable for storing received data

void setup()

\{ Serial.begin(9600); //Sets the data rate in bits per second (baud) for serial data transmission pinMode(13, OUTPUT); //Sets digital pin 13 as output pin \}

$\operatorname{void} \operatorname{loop}()$ 
$\{$ if $($ Serial.available ()$>0) / /$ Send data only when you receive data:

$\{$ data $=$ Serial.read ()$; / /$ Read the incoming data and store it into variable data

Serial.print(data); //Print Value inside data in Serial monitor

Serial.print("\n"); //New line

if (data $==$ '1') //Checks whether value of data is equal to 1

digitalWrite(13, HIGH); //If value is 1 then LED turns ON

else if $($ data $==$ ' 0 ') //Checks whether value of data is equal to 0

digitalWrite(13, LOW); //If value is 0 then LED turns OFF \} \}

\section{Conclusion}

The product thus developed is fully operational and gives desired motion. It is being tested in a room which results in successful outcome. Manually operated floor cleaning machine is an alternative for an automated floor cleaning machines during power crisis. Body is machine operated to achieve dry and wet Manual effort and time is reduced less as cleaning and polishing can be done at same time. Design is very simple and easy fabrication. Overall the concept is very much helpful and there is scope of a lot of development in mechanical parts. The optimization will continue till achieving the best one. Overall the project is successful to its intent and will definitely change the era robotics and floor cleaning. In the automation part the algorithm are designed to give $90 \%$ efficiency which is too high in current scenario. The development can be made in the field of sensing.

\section{Future Scope}

The scrubber design should be modified in future because the current design has few problems. Few of those are the motor is not detachable and the high rpm leads to vibration of the whole system. If these features will be modified, this will work well. The development can be made in the field of sensing and to detect as well as move in the direction of dust and thus resulting in better cleaning of floors. Monitoring, self-charging, lighter body weight and to set alarm on/off time manually are the future scope of this paper.

\section{References}

1. www.eeweb.com/project/floor-cleaning-machine-remote-controlled

2. www.standardsuniversity Clear

3. Mahoney.R, Service Robotics.org/wp-content/.../Smart-Floor-Cleaning-Robot-

4. Case Studies in Silicon Valley Silicon Valley Robotics Technical Report, 2015

5. Jagdeesh Chandra A.P, Venugopal C.R, Novel Design solutions for remote access acquire and control of Laboratory experiments on DC machines, IEEE transaction on instrumentation and measurement, 61, 2, 2012.

6. https://www.youtube.com/watch?v=UAVKLUAV7-E

7. Liu, Kuotsan1, Wang, Chulun, A Technical Analysis of Autonomous Floor Cleaning Robots Based on US Granted Patents, European International Journal of Science and Technology, 2, 7; 2013. https://www.researchgate.net/publication/299372806_Floor_Cleaning_Robot_with_MobileApp_or_Autonomous 